XV.

Aus dem pharmakolog. Institut der Deutschen Universität in Prag.

Experimenteller Beitrag zum 0xalsäurestoffwechsel.

Bemerkungen zur gleichlautenden Arbeit Dr. Zd. Tomaszewski's, Bd. 7, S. 215 dieser Zeitschrift, 1909.

Von

\title{
Julius Pohl.
}

In der angeführten Arbeit erörtert T. wieder einmal die Frage der Oxydation der Oxalsäure durch den lebenden Organismus, ihre Beziehungen zur Harnsäure etc. und kommt hierbei auch auf ältere Versuche von mir, die sich mit demselben Thema befassen, zu sprechen. Schon an die eingeschlagene Methode, die alte Neubauer'sche, knüpft er eine absprechende Kritik: sie soll mit $25 \mathrm{pCt}$. Verlust arbeiten. Dem gegenüber sei hervorgehoben, dass nach Huppert, Analyse des Harns, 10. Aufl. S. 788 , dieselbe auf 2,5 pCt. genau ist.

Die Methode ist somit zur Oxalsäurebestimmung im Harn genügend verlässlich und die mit ihr erhaltenen Resultate bleiben zu Recht bestehend.

Was nun die Frage der Oxydationsfähigkeit der Oxalsäure im thierischen Organismus anbelangt, so gehe ich nur mit Widerstreben daran, für dieses schon längst entschiedene Problem neuerlich das Wort zu ergreifen.

$\mathrm{Zu}$ den älteren Versuchen von Gaglio und mir hat noch Rotter ${ }^{1}$ ) hierfür folgende Belege geliefert:

Injicirt wurden Hunden je $0,006,0,01,0,048$ Oxalsäure und wiedergefunden wurden $0,0069,0,01,0,038$. Ferner hat seither $\mathrm{F}^{2}$ aust $\left.{ }^{2}\right)$ neun Versuche veröffentlicht, bei denen er $95,06 \mathrm{pCt}$. der injicirten Oxalsäure gefunden hat. Obwohl also das Resultat weiterer Versuche klar vorauszusehen war, liess ich dennoch neuerlich einen derartigen Versuch durch meinen Demonstrator, H. Schwarzbach ausführen.

\section{Versuch.}

$10 \mathrm{ccm}$ einer Natr.-Oxal.-Lösung enthalten Oxalsäure gleich 0,0212 $\mathrm{CaO}$. Von derselben lüsung werden einem kleinen Hunde $10 \mathrm{ccm}$ subcutałn

1) Arch. f. experim. Path. u. Pharmakol. Bd. 42. S. 379. 1899.

2) Ebendas. Bd, 47. S. 237. 1900. 
injicirt. Aus dem Harn der nächsten 2 Tage wird nach obigem Verfahren Oxalsäure gewonnen gleich $0,0218 \mathrm{CaO}$. Hiervon sind die schwankenden 2-3 mg $\mathrm{CaO}$ betragenden Normalwerthe abzuziehen.

Wir können also ruhig resumiren, dass Oxalsäure thatsächlich zu den im Organismns unangreifbaren Substanzen gehört; findet man nach Darreichung grösserer Dosen Verluste, so ist immer an die Möglichkeit ihrer Ablagerung ais oxalsaurer Kalk zu denken; Versuche mit Darreichung vom Darm aus, wie sie z. B. Autenrieth und Barth angestellt haben, können natürlich für eine von Mikroorganismen zersetzbare Substanz nichts beweisen.

Was nun die Frage der Oxalsäurebildung aus Harnsäure anbelangt, so kommt T. mit dieser Ansicht wahrlich post festum. Für den thierischen Organismus ist in meinem Institute der partielle Uebergang der Harnsäure in Allantoin ebenso sicher nachgewiesen (Wiechowski), wie die Unzerstörbarkeit des entstandenen Allantoins (Poduschka), und auch für den Menschen ist, wieder durch Wiechowski, die Unangreifbarkeit der Harnsäure nachgewiesen: da es für den Menschen eine Urikolyse kaum giebt, so muss für die Oxalsäureausscheidung bei demselben nach einer anderen Quelle gesucht werden. Die Nothwendigkeit für den Zustand der Oxalurie, für die Bildung von Oxalatconerementen Aufklärung zu suchen, soll nicht geleugnet worden; doch sprechen auch diese Affectionen weit eher für die Unzerstörbarkeit als für die Angreifbarkeit der Oxalsäure.

T. bringt sodann Versuche mit Organaufgüssen, die cine Zerstörung bereits gebildeter oder hinzugefügter Oxalsäure beweisen sollen. Fr verwendet hierbei ein Verfahren, das in einem Controlversuch bereits 20,4 pCt. Verluste giebt und findet mit demselben, dass Niere und Leber ein Zerstörungsvermögen für Oxalsäure besitzen. Auch hierüber habe ich durch H. Schwarzbach einen Versuch ausführen lassen, der folgendermaassen verlief:

\section{Versuch.}

Zwei normale Kaninchenlebern werden zu Brei verrieben und in 3 gleiche Theile à $30 \mathrm{~g}$ getheilt. In I wird die präexistente Oxalsäure bestimmt, in II sofort nach Hinzufügen einer Lösung von $10 \mathrm{ccm}$ oxalsaurem Natr. $=0,047 \mathrm{CaO}$ und in IlI dasselbe nach 24 stündigem Verweilen im Brutofen bei $38^{\circ}$. Die Proben werden auscoagulirt und in den Filtraten die Oxalsäure bestimmt. In I $0,0017 \mathrm{~g} \mathrm{CaO}$, in II 0,0495 , somit nach Abzug von $\mathrm{I}=0,0478$, in III 0,0476 nach Abzug von $\mathrm{I}$ $=0,0459 \mathrm{CaO}$ gewonnen. $\mathrm{Da}$ von $47 \mathrm{mg} 46$ wiedergefunden wurden, so ist, was ganz mit den Versuchen am lebenden Thiere übereinstimmt, nichts von der Substanz zerstört worden.

$\mathrm{T}$. erinnert sodann an die in der Jiteratur geäusserte Anschauung, dass der Abbau der Harnsäure in den Organen über die Glyoxylsäure gehen könnte. Auch diese Auffassung ist irrthümlich, denn zwischen der Harnsäure und der Glyoxylsäure bestehen ebensowenig Beziehungen in vivo, als zwischen der Oxalsäure und der Harnsäure. Es hat Adler ${ }^{1}$ )

1) Arch. f. experim. Path. u. Pharmalrol. Bd. 56. S. 207. 1907. 
1907 nachgewiesen, dass die Glyoxylsäure durchaus nieht indifferent ist, dass sie nach grösseren Dosen ein charakteristisches Herzphänomen auslöst, den Pulsus alternans ${ }^{1}$ ), und dass sie ausserdem rasch in Oxalsäure übergeht. Nun ist es für den Hund erwiesen, dass die im Harn gefundenen Werthe für Harnsäure plus Allantoin der Quantität injieirter Harnsäure entsprechen, ferner ist sichergestellt, dass vom Menschen 80 bis 90 pCt. injicirter Harnsäure unverändert ausgeschieden werden ${ }^{2}$ ). Gerade in dieser Richtung ist eine erfreuliche Bestätigung aus derselben Klinik, an der T. arbeitete, mitgetheilt worden, indem Benczur ${ }^{3}$ auch beim Gichtkranken das gleiche Ausscheidungsvermögen, wie es beim Normalen festgestellt worden ist, fand. Bei dieser Sachlage noeh immer in der Harnsäure einen Ursprungskörper für pathologische Producte zu suchen, erscheint völlig unbegründet.

Bisher ist es, in Uebereinstimmung mit der von mir vertretenen These der Unzerstörbarkeit der Oxalsäure immer gelungen, in jenen Fällen, wo Oxalsäure intermediär aus anderen Vorstufen oder Verbindungen entsteht, dieselbe nachzuweisen. Ich erinnere an die Bildung von Oxalsäure nach Darreichung von Alloxan, Glycol, nach Glyoxylsäure. Eine Ausnabme biervon macht nach den vorhandenen literarischen Angaben die Oxalursäure resp. Parabansäure. Während Luzzato), der in Ammoniak gelöste Parabansäure, also Oxalursäure an den Hund verabreichte, aus einem Versuch Umwandlung in Oxalsäure und vollständige Oxydationderselben deducirt, giebt Coppola ${ }^{5}$ ) an, dass dieselbe unverändert in den Harn übergeht. Das gegenseitige Verhältniss dieser Substanzen wird aus folgenden Formelbildern klar:

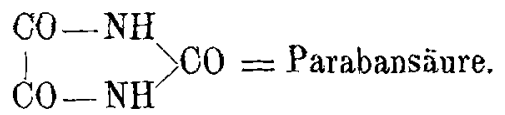

$$
\begin{aligned}
& \mathrm{NH}_{2} \cdot \mathrm{CO} \cdot \mathrm{NH} \cdot \mathrm{CO} \cdot \mathrm{COOH}=\text { Oxalursäure. } \\
& (\mathrm{COOH})_{2}=\text { Oxalsäure. }
\end{aligned}
$$

Bei dieser Sachlage stellte ich mit der Parabansäure einige Versuche an, von denen hier einer angeführt werden möge. Zura Verständniss des Folgenden sei erwähnt, dass Parabansäure resp. Oxalursäure durch Kochen mit Ammoniak quantitativ in Oxalsäure übergeht. Die Möglichkeit, Parabansäure neben Oxalsäure im Harn quantitatív nachzuweisen, bernht auf der Thatsache, dass in neutraler bis schwachsaurer Lösung Parabansäure mit neutralem Bleiacetat nicht fallt, während Oxalsäure ansgefällt wird. Ich theile deshalb den Harn in zwei Theile, bestimme in einem Theile die Gesammtsumme des Oxalats nach Aufkochen mit Ammoniak auf kochendem Wasserbade (somit die Summe aus nativer Oxalsäure

1) Siehe auch Starkanstein. Diese Zeitschr. Bd, 4. S.681. 1907.

2) Siehe die letzte zusammenfassende Darstellung bierüber bei W. Wiech ow ski, Biochem. Zeitschr. Bd. 25. S. 431.

3) Diese Zeitschr. Bd. 7. S. 338.

4) Zeitschr. f. physiol, Chem. Bd. 37. S. 243.

5) Cit. nach Fraenkel, Arzneimittelsynthese. II. Aufl. 
plus der aus der Parabansäure entstandenen), in einer zweiten Probe, die nach Ausfällung mit Bleiacetat und Kochen mit Ammoniak nachweisbare Oxalsäure (entspricht der Parabansäure).

III. Versuch. Beleg für die Zulässigkeit dieses Verfahrens.

100 cem Normalharn liefern nach Kochen mit $\mathrm{NH}_{3}$ - Parabansäure + Oxalsäure . . . . . . . . . . . . . . 0,028 $\mathrm{CaO}$

Oxalsäure allein . . . . . . . . . . . . . . 0,0017

Somit für physiologische Oxalursäure resp. gepaarte

Oxalsäure . . . . . . . . . . . . . 0,0011 g

$10 \mathrm{ccm}$ einer Lösung von Parabansäure in Wasser gelöst

liefert Oxalsäure . . . . . . . . . $=0,0373 \mathrm{CaO}$

$100 \mathrm{ccm}$ Harn $+10 \mathrm{ccm}$ derselben Parabansäurelösung

geben, nach Behandlung mit Bleiacetat, Oxalat $=0,0385 \mathrm{CaO}$

Somit nach Abzug obiger Differenz von . . . . . 0,0011 wiedergefundene Parabansäure . . . . $=0,0374$

\section{Versuch. Hund $6840 \mathrm{~g}$.}

Vortag: $330 \mathrm{ccm}$ Harn mit Oxalsäure. . . . . $=0,0079 \mathrm{~g} \mathrm{CaO}$ Subcutan wird vorsichtig mit $\mathrm{Na}_{2} \mathrm{CO}_{3}$ neutralisirte Para-

bansäure injicirt. . . . . . . . . . . $=0,0525 \mathrm{~g} \mathrm{CaO}$

An den 2 nächsten Tagen 975 Harn mit . . . . 0,0714 g CaO

Abzug für 2 Tage . . . . . . . . . . . $0,0158 \mathrm{~g} \mathrm{CaO}$

Plus über die Norm. . . . . . . . . $\frac{.0,0556 \mathrm{~g} \mathrm{CaO}}{0.0350 \mathrm{~g}}$

An Parabansäure am 1. Tage gefunden . . . . . $0,0350 \mathrm{~g} \mathrm{CaO}$

Am 2. Tage keine Parabansäure im Bleiacetatfiltrat. Für

Oxalsäure verbleibt . . . . . . . . . $0,0206 \mathrm{~g} \mathrm{CaO}$

Dass der Werth Parabansäure + Oxalsäure an den zwei Versuchstagen etwas grösser ist, als der injicirten Parabansäure entspricht $(0,0556$ gegenüber 0,0525 ), ist wohl ungezwungen aus der Zunahme des Harnvolumens und der damit parallel gehenden Steigerung der Oxalatwerthe zu erklären.

Der Versuch lehrt somit, dass ein Theil der injicirten Parabansäure nnverändert in den Harn übertritt, der fehlende wird als Oxalat ausgeschieden. Zwei weitere Versuche verliefen ganz gleichsinnig. Es steht somit das Schicksal der Parabansäure resp. Oxalursäure in vollem Einklang mit unseren Laboratoriumserfahrungen, dass Substanzen, die intermediär Oxalsäure bilden, dieselbe durch den Harn unverbrannt ausscheiden. 